January 3,1893 , not having.opened the box for some days, I made an examination. The egg was in its former position, so far as I could tell, but the shell was split on one side and the young Peripatus had escaped. This young Peripatus was found lying dead on the glass floor of the hatching box, $25 \mathrm{~mm}$. distant from the shell. It must have crawled off the rotten wood and along the glass to the position in which it was found. It was only ahout $5 \mathrm{~mm}$. in length, so that, even assuming that it moved in a perfectly stıaight line, it must have crawled tor a distance five times its own length. To the naked eye the young animal appeared of a pale greenish colour. It could not have been dead for very many days, but decomposition had already set in, and the animal was stuck to the glass on which it lay. It was impossible to remove it without considerable injury, but I ultimately succeeded in mounting it in Canada balsam, and it is impossible, even in its present condition, to doubt that it really is a young Peripatus, for the characteristic jaws and claws are well shown. I also mounted the ruptured egg-shell, and found that the characteristic sculpturing on the outside was still clearly visible.

This egg, then, hatched out after being laid for about seventeen months (from about July I89 to about the end of December 1892). I cannot believe that under natural conditions the embryos take so long to develop. At any rate it now appears certain that the larger Victorian Peripatus lays eggs which may hatch after a lapse of a year and five months.

The University of Melbourne, February.

$$
\text { ARTHUR DeNDY. }
$$

\section{A Simple Rule for finding the Day of the Week corre- sponding to any given Day of the Month and Year.}

A RULE was lately mentioned to me by a friend for finding, almost by inspection, the day of the week for any given year and day of any month in that year, during the present century. The basis of the rule is so obvious, when once the rule is stated, as to require no demonstration, but it struck me as so ingenious as to be worth while communicating it to you in case you deemed it worthy of insertion. I also append a very easy method of extending the rule to any date subsequent to the introduction of the Julian intercalation either in the past or future, except indeed for the eighteenth century, in which the introduci inn of the new style requires a special treatment.

The nineteenth century rule above alluded to is this. Each of the 12 montbs has its special numerical constant, thus:-

$$
\text { Jan. Feb. Mar. Ap. May June July Aug. Sept. Oct. Nov. Dec. }
$$

Write down four columns thus

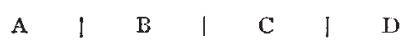

Under A enter day of month, under B constant for that month, under $C$ year of century, under D greatest multiple of 4 in the year of century.

Add together the numbers under these heads, divide by 7 , and the remainder is day of week; except that in Leap Year I must be subtracted for any day before February 29.

$$
\text { Example.-June 18, I815 (Battle of Waterloo) :- }
$$

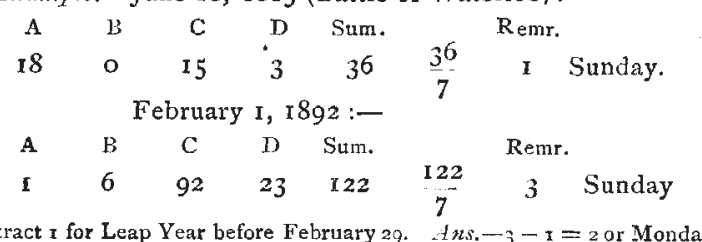

Subtract $x$ for Leap Year before February 29. $4 n s-3-1=2$ or Monday.

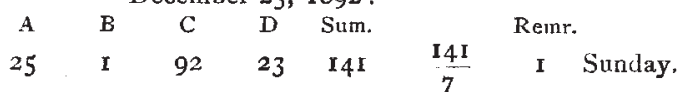

To extend the rule to any future century, we have only to alter the monthly constants, adding 5 to each for each added century after the present, and I for each century, an exact multiple of 4 , in the interval.

Thus for the thirty-first century. Number of added centuries is 12 , and there are 3 centuries, succeeding multiples of 4 (twenty-first, twenty-fifth, and twenty-ninth). Therefore add $5 \times 12+3=63$, or omitting multiples of 7 , add 0 .

No. I 222 , VOL. 47]
Hence, constants for thirty-first century are the same for the present century.

New Year's Day, 300I,

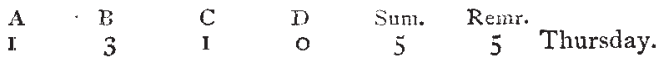

For centuries anterior to the eighteenth we must first of all find by special method what the monthly constants would have been throughout the eighteenth century without the change of style, and then subtract 6 for each century short of the eighteenth.

It may easily be seen that the constants throughout the eighteenth century would have been without change of style.

Jan. Feb. Mar. Ap. May June July Aug. Sept. Oct. Nov. Dec.

For the eleventh century subtract $7 \times 6$ or 42 , i.e. since this is multiple of 7 subtract $o$, and we get the same repeated.

For the seventeenth subtract 6 , and remember that when the result is negative we must replace it by the defect of the corresponding positive number from 7 , and we get

$$
\begin{array}{llllllllllll}
3 & 6 & 6 & 2 & 4 & 0 & 2 & 5 & \text { I } & 2 & 5 & \text { I }
\end{array}
$$

Example.-Battle of Hastings, Oct, 14, 1066.

$$
\begin{aligned}
& \text { A B C D Sum. Remr. }
\end{aligned}
$$

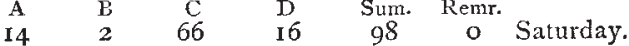

Execution of Charles I., Jan. 30, 1649,

$$
\begin{aligned}
& \text { A B } C \text { D Sum. Remr. }
\end{aligned}
$$

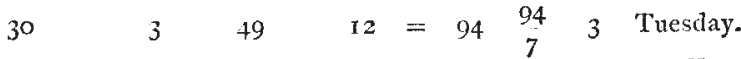

$$
\begin{aligned}
& \text { H. W. W. }
\end{aligned}
$$

\section{"Roche's Limit."}

With reference to Prof. G. H. Darwin's notes (NATURE, March $16, \mathrm{p}, 460$ ) on the investigations of $M$. Roche as to the smallest distance from its primary at which a satellite can exist, does not the distance given-viz. 2.44 times the radius of the primary-refer to the case of the satellite having the same density as its primary? In Note 3 Prof. Darwin warns the reader that Roche's limit depends, to some extent, on the density of the planet. Suppose the density of the planet to remain the same while that of the satellite is taken at double. In this case the tidal or differential influence of the planet on the two halves of the satellite will have doubled, while the gravitational attraction of the two halves of the satellite on each other will have become fourfold ; and generally, the power of the planet to pull the satellite asunder will be inversely as the density of the satellite, and directly as the density of the planet.

An alteration of the size of the satellite does not much affect the question, because both forces are thereby equally altered, so long as the satellite is very small in comparison with its distance from the planet.

Seeing that the tidal or differential influence of a planet on its satellite is inversely as the cube of their distance apart, perhaps it would be correct-as far as gravitational influence alone is concerned-to state the limit at which a satellite can exist as being equal to $2.44 \mathrm{R} \times\left(\frac{\mathrm{D}}{d}\right)^{\frac{1}{3}}$ where

$$
\begin{aligned}
\mathrm{R} & =\text { the radius of the planet, } \\
\mathrm{D} & =\text { the density of the planet, } \\
d & =\text { the density of the satellite. }
\end{aligned}
$$

As an interesting case of the same problem from a different point of view, suppose two very small equal spheres in contact, and a third much larger sphere placed in line with their centres, all three having the same density; then, when the distance of the point of contact of the small spheres from the centre of the large one is 2.52 times the radius of the large one, the attraction of the two small spheres for each other just balances the differential influence of the large one tending to draw them asunder. The effects of varialion in density and size being the same in this case as in the former.

It would probably be interesting to many of your readers to have Prof. Darwin's views as to whether it is a reasonable supposition that a small satellite, such as Jupiter's fifth, is likely to have the same density as Jupiter; and whether the meteorites forming Saturn's ring are likely to be of so small density as 
Saturn; as it would appear that without making some such supposition, no definite limit can be fixed.

Applying this supposition to the sun, with reference to meteoric swarms, we have $2{ }^{\circ} 44$ times the sun's radius, taken at 433,000 miles, or $1,056,520$ miles as the distance at which the sun would prevent the meteors coalescing to form a planet. In Note 3 Prof. Darwin states this at one-tenth of the carth's dis tance from the sun, probably by inadvertence.

G. R.

\section{The Ordnance Survey and Geological Faults.}

In view of the re-survey of the United Kingdom, it seems to me that if the officers of the Survey were directed to take special notice of the levels of the former survey on both sides of great geological faults, and to compare these levels now so as to ascertain if any appreciable relative change had taken place during the forty or fifty years since the first survey, valuable information as to the motion of these faults, if any, might be obtained.

This idea is mainly suggested to me by the fact that in this neighbourhood a great fault intersects the Old Red Sandstone close ts its contact with the Highland schists, it has been traced from Stonehaven on the east coast to Loch Lomond on the west, and seems to give remarkable evidence of being, at least to a certain extent, in motion. The village of Comrie, famous for its " earthquakes," is situated on this fault, and the "earthquakes" are as lively as ever. In the valley of Strathmore farmhouses placed in the proximity of this great dislocation are, or were, celebrated for being "haunted," on account of the noises and tremors by which the inhabitants are from time to time alarmed.

Most, if not all, British "earthquakes" have been, I think, wisely attributed to similar cau-es.

Of course fifty years is a very minute part of the history of one of these old faults, but if the data of the Ordnance Survey be so accurate as is usually supposed, some trace of shifting might possibly be discovered if the necessary observations were made. Newport, Fife, March 18.

JAS, ITRRHAM,

\section{The Discovery of the Potentia!}

Mr. E. J. Rourh has lately published a most valuable "Treatise on Analytical Statics." I quote from the second volume, $p .17$, the following note :-

"The earliest use of the function now called the potential, is due to Legendre in 1784 , who refers to it when discussing the attraction of a solid of revolution. Legendre, however, expressly ascribes the introduction of the function to Laplace, and quates from him the theorem connecting the components of attraction with the differential coefficients of the function. The name, Potential, was first used by Green," etc.

From this note it appears that the discovery of the potential must be attributed to Laplace. This is a wrong opinion, and some fifteen years ago Baltzer proved that the introduction of the function is due to Lagrange ("Zur Geschichte des Potentials," in Gournal fiir die reine und angetvandte Mathematik, vol. 1xxxvi. p. 213, I878). Some historical documents in favour of Lagrange's priority have been found, by the writer of these lines, in Todhunter's "History of the Mathematical Theories of Attraction and the Figure of the Earth," and collected in a note at the end of vol. i. of his work, "Il Problema Meccanico della Figura della Terra" (Torino, I880), where a full account of the early history of the potential is given, with numerous bibliographical indications.

Private Docent in the University of Turin, March 21 .

THE historical note on p. I 7 of my "Statics" is chiefly founded on the statements in Todhunter's "History," and in Thomson and Tait's "Natural Philosophy." The references to these two writers are given in the note. Both Dr. Todhunter and Lord Kelvin ascribe the introduction of the function for gravitation to Laplace, and assert that the name of "Potential" was first given to it by Green. My own reading, though not so extensive as theirs, had not led me to form any different opinion. In Nichol's "Cyclopædia of the Physical Sciences" the first introduction is given as due chiefly to Legendre, Lagrange, Laplace, and Poisson. In Chambers's "Cyclopæedia" Laplace's name alone is mentioned. Baltzer, as cited by $\mathrm{Mr}$. Bianco, mentions the use of the function by Lagrange in the M'm. de Berlin, 1777 . This is earlier than the memoir of Legendre, but as Legendre assigns NO. I 222 , VOL. 47] the introduction of the function to Laplace, it is difficult to compare the dates. I am at present unable to refer either to the memoir of Lagrange or to the treatise of $\mathrm{Mr}$. Bianco.

E. J. RovTh.

\section{Van't Hoff's "Stereochemistry."}

"nit review of the above by "F. R. J." in Nature, p. 436 , raises some important points in connection with this peculiarly fascinating branch of chemical science. In referring to the recent ingenious and attractive theory of $P$. A. Guye, that the numerical value of optical activity is dependent upon the relative masses of the four groups attached to the asymmetric carbon atom, and which carries with it the corollary that if two of these four groups are of equal mass the rotatory power will cease, your reviewer states that Guye "was unable to verify this view in all strictness." I think, however, that he hardly emphasises sufficiently that this important corollary has in every case, when put to the test of direct experiment, broken down. As far as I am aware, there is not a single instance of an asymmetric carbon atom attached to four groups quatitatively distinct, being found optically inactive in consequence of two of those groups being quantitatively equal in mass. Indeed some such substances are not merely active but powerfully so. The reviewer considers that this inadequacy of Guye's theory is palliated by the alleged fact that the amount of rotatory power of the esters of an active acid is determined by the weight of the alkyl-group. This point, which is one of the cardinal pillars of Guye's theory, I have recently put to the test of actual experiment, by measuring the rotatory power of a number of the esters of active glyceric acid, which have been prepared by Mr. J. MacGregor and myself. In this investigation we found the most cxtraordinary verification of Guye's theory, as far as the optical properties of the normal series of methyl, ethyl, and propyl glycerates were concerned; with the appearance of isomerism, however, this recrularity ceases, thus the isopropyl glycerate has a markedly lower rotation than the normal one, whilst the normal and secondary butyl compounds have a lower rotation than the isobutyl ester. Nor are these differences consistently explicable by taking into consideration the interatomic distances, as measured by atomic volume, for the molecular volume of the normal propyl glycerate with its greater rotation is less than that of the isopropyl compound with its smaller rotation, whilst the molecular volumes of the isobutyl and secondary butyl glycerates are almost exactly equal, although the rotation of the former is much greater than that of the latter.

The reviewer, in referring to the rotation exhibited by the salts of active acids, states that in the case of tartaric acid all the salts "display in solution the same rotatory power, irrespective of the atomic weight of the metal," and is apparently satisfied that " the clue to this anomaly is furnished by the electrolytic theory of Arrhenius," according to which "it is the ion $\mathrm{CO}_{3}(\mathrm{CHOH})_{3}$. $\mathrm{CO}_{2}$ which is alone responsible for the rotation." The reviewer has in this endorsed the method of special pleading adopted by the advocates of this theory, in which the metallic tartrates have been summoned as witnesses, whilst only the testimony of those favourable to the theory has been admitted. Thus one of the commonest of the metallic salts of tartaric acid--tartar emetichas a rotation which differs entirely from that of the other tartrates, and thus conclusively negatives the dogma that the rotation of the solutions of metallic salts is independent of the particular metal which has replaced the hydrogen of the acid. Fresh light has been thrown on this point in the course of an investigation, which I have recently carried out with $\mathrm{Mr}$. Appleyard on the rotatory power of the metallic salts of active glyceric acid, and which has shown that the specific rotatory power of the glyceric acid has one value when deduced from the rotations of its alkaline salts (lithium, ammoniuın, sodium, "and potassium), another value when deduced from the salts of the alkaline earths (calcium, strontium, and barium), sand a third from the salts of the magnesium group of metals (magnesium, zinc, and cadmium). Now it so happens that almost the only salts of tartaric acid which have had their rotation determined are those of the alkaline metals. which also in the case of glyceric acid yield practically the same rotation. Hence if only the rotations of the alkaline glycerates had been determined, the same erroneous conclusion would have been arrived at concerning the rotation of glyceric acid. Whatever may be the ultimate interpretation put upon these new results, and I prefer for the present to ab- 